\title{
Chronic pneumonia due to Klebsiella oxytoca mimicking pulmonary tuberculosis
}

The authors declare no financial disclosure

\begin{abstract}
Klebsiella species infrequently cause acute community acquired pneumonia (CAP). The chronic form of the disease caused by K. pneumoniae (Friedlander's bacillus) was occasionally seen in the pre-antibiotic era. $K$. oxytoca is a singularly uncommon cause of CAP. The chronic form of the disease caused by $K$. oxytoca has been documented only once before. A 50 -year-old immunocompetent male smoker presented with haemoptysis for 12 months. Imaging demonstrated a cavitary lesion in the right upper lobe with emphysematous changes. Sputum stains and cultures for Mycobacterium tuberculosis were negative. However, three sputum samples for aerobic culture as well as bronchial aspirate cultured pure growth of $K$. oxytoca. A diagnosis of chronic pneumonia due to $K$. oxytoca was established and with appropriate therapy, the patient was largely asymptomatic. The remarkable clinical and radiological similarity to pulmonary tuberculosis can result in patients with chronic Klebsiella pneumonia erroneously receiving anti-tuberculous therapy.
\end{abstract}

Key words: cavitary lesions, chronic necrotising pneumonia, imaging, Klebsiella oxytoca, Mycobacterium tuberculosis

Pneumonol Alergol Pol 2015; 83: 383-386

\section{Introduction}

Klebsiella, an aerobic gram-negative bacillus, is a rare but well established cause of community acquired pneumonia (CAP), especially in the pre-antibiotic era [1]. This organism continues to be an infrequent source of CAP accounting for $1-5 \%$ of the patients [2-4]. However, two studies have documented Klebsiella as a causative organism of CAP in as high as 12 and $22 \%$ of the patients respectively [5, 6]. Although, an uncommon cause of CAP, hospital acquired pneumonia caused by Klebsiella is fairly frequent and can be life threatening $[7,8]$.

CAP due to K. pneumoniae usually has an acute presentation resembling that of pneumococcal lobar pneumonia, occurring almost exclusively in debilitated subjects [5]. Rarely, pneumonia caused by Klebsiella species can manifest as a chronic disease, which can last for weeks or months. This singularly uncommon form of the disease may be seen in elderly subjects and the presentation is strikingly similar to that of pulmonary tuberculosis [1, 9-11]. In high tuberculous prevalent areas, this unusual clinical entity can often be misdiagnosed and treated for tuberculosis while lung damage continues to occur [11]. Other important conditions that can simulate pulmonary tuberculosis radiologically include non-tuberculous mycobacterial infections, other bacterial pneumonias especially pulmonary nocardiosis, fungal infections, allergic bronchopulmonary aspergillosis and lung malignancies [12]. Although K. pneumoniae (Friedlander's bacillus) is the most commonly cultured organism, three other species viz. K. oxytoca, $K$. rhinoscleromatis and $K$. ozaenae can also be responsible infrequently [13]. K. oxytoca has rarely been implicated

Address for correspondence: Ashok Shah, Department of Respiratory Medicine, Vallabhbhai Patel Chest Institute, University of Delhi, Delhi 110007 , India, e-mail: ashokshah99@yahoo.com

DOI: 10.5603/PiAP.2015.0061

Received: 15.04 .2015

Copyright (C) 2015 PTChP

ISSN 0867-7077 
as a cause of CAP. The reports available in the literature suggest that CAP due to this organism has an acute manifestation. This was highlighted by a study from Switzerland which reported $12(12.4 \%)$ patients with CAP requiring hospitalisation due to Klebsiella species which included two caused by K. oxytoca [5]. The chronic form of the disease caused by K. oxytoca has been documented only once previously in an 82-year-old female from Canada who presented with a non-resolving pneumonia of 9 months duration [14].

The paucity of the literature on the subject prompted us to report a middle-aged man with a 30-pack-year history of smoking who presented with chronic necrotising pneumonia of 12 months duration caused by $K$. oxytoca.

\section{Case report}

A 50-year-old male, non-diabetic, HIV-negative, a farmer by occupation, was referred to our Institute for evaluation of haemoptysis of 12 months duration. Haemoptysis was intermittent with 10 to 12 episodes over the past year. Blood was often mixed with sputum but recently he had experienced two episodes where he had expectorated about $500 \mathrm{~mL}$ of frank blood, which prompted the referral. For the preceding two months this was associated with cough and expectoration of approximately four to five teaspoonful of non-foul smelling muco-purulent sputum. He had smoked 30 pack-years before quitting one year ago with the first episode of haemoptysis. He had received several short courses of oral antibiotics for this complaint without relief. However, he had not received anti-tuberculous therapy. On presentation, general physical and respiratory system examinations were unremarkable.

Complete blood counts, ECG, urine analyses and renal as well as hepatic function test results were within normal limits. Chest apicogram done on presentation showed the presence of a small cavity in right upper zone with increased lung markings as compared to the left upper zone (Fig. 1). A high resolution computed tomography (HRCT) of the thorax demonstrated thick walled cavity in apical segment of right upper lobe surrounded by fibrosis and centrilobular nodules while emphysematous bullous area was seen in left upper lobe (Fig. 2). Paraseptal emphysema was also visible bilaterally along with centrilobular emphysema in the left lung. Small nodular lesions were also visible in left lower lobe. However, spirometry with reversibility was within normal limits with post-bronchodilator $\mathrm{FEV}_{1} / \mathrm{FVC}$ ratio of 0.77 .

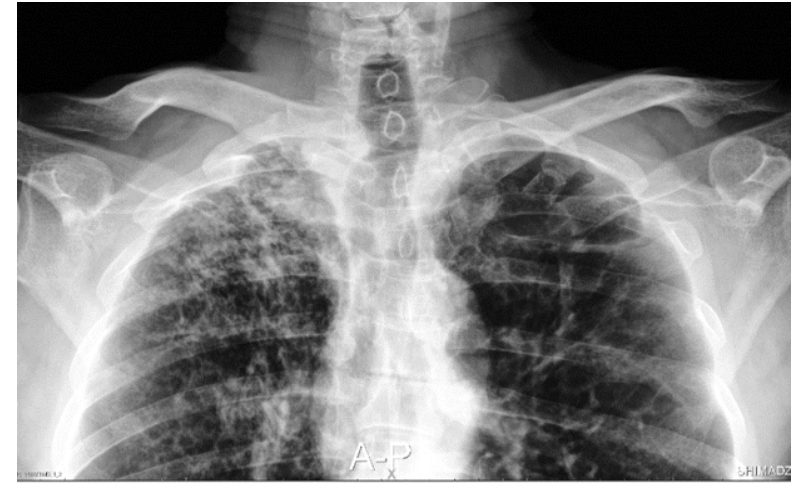

Figure 1. Chest apicogram done on presentation showed the presence of a small cavity in right upper zone with increased lung markings as compared to the left upper zone

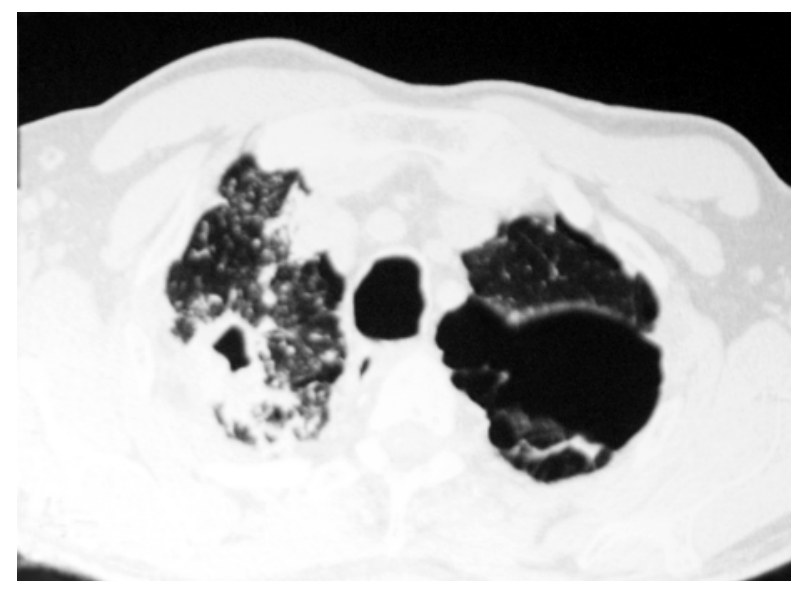

Figure 2. HRCT of the thorax demonstrated thick walled cavity in apical segment of right upper lobe surrounded by fibrosis and centrilobular nodules while emphysematous bullous area was seen in left upper lobe

Several stains for acid-fast bacilli (AFB) done prior to presentation were negative. Three sputum smears for AFB and cultures for $\mathrm{Myco-}$ bacterium tuberculosis done at presentation were also negative. K. oxytoca was isolated as a pure growth in sputum culture performed for aerobic bacteria other than M. tuberculosis. The organism was cultured in the initial sample and the subsequent two samples also and was sensitive to almost all common antibiotics. Fiberoptic bronchoscopy visualised a normal bronchial mucosa. Cytological examination of the bronchial aspirate smear showed benign columnar epithelial cells, moderate infiltration of lymphocytes and few macrophages with no malignant cells. Aerobic culture of the aspirate also yielded pure growth of $K$. oxytoca. Stains and cultures for $M$. tuberculosis or other aerobic organisms continued to be negative. 
A diagnosis of chronic pneumonia caused by K. oxytoca was made. Although the spirometry was within normal limits, the patient had a 30-pack-year history of smoking and the CT picture was suggestive of emphysema. The patient had never experienced any symptoms suggestive of exacerbation of chronic obstructive pulmonary disease (COPD) nor had he ever received inhaled/oral corticosteroids or any other therapy. Based on the culture sensitivity report, the patient received antimicrobial therapy with oral amoxicillin/clavulanic acid (500 + 125 mg 8 hourly) along with intravenous gentamicin (160 mg 12 hourly) for a fortnight. With this, the patient experienced marked symptomatic improvement and within 2 weeks, haemoptysis was largely abolished. The patient was relatively symptom-free and after 3 months he was lost to follow-up.

\section{Discussion}

Pneumonia due to Klebsiella generally occurs in elderly people with risk factors including alcoholism, diabetes mellitus, smoking, co-morbidities such as COPD and other debilitating illnesses [6, 13]. Of the 2,776 patients hospitalised for CAP in 1991 in two counties in Ohio, Klebsiella species were implicated in 29 (1\%) [3]. The retrospective study from Switzerland [5] in 293 hospitalised patients with bacterial pneumonia, specific bacteriological aetiologies were found in 97 (33.1\%). Twelve (12.4\%) of these patients were due to Klebsiella species [5]. A prospective study in 70 patients with CAP from India reported aetiological confirmation in 53 (75.6\%), 12 (22.6\%) of whom were caused by K. pneumoniae [6].

K. pneumoniae is usually the causative species but $K$. oxytoca as a potentially significant respiratory pathogen was first recognised in a retrospective study in 1983. This study found that of the 4,800 patients with respiratory complaints, 110 sputum samples and one blood culture were positive for Klebsiella species. Of these, K. oxytoca was isolated in 11 samples from 11 patients including the one from blood culture. Four of these 11 patients had lobar pneumonia while one had bronchopneumonia, five had acute exacerbations of chronic bronchitis and one had an acute respiratory tract infection superimposed on cryptogenic fibrosing alveolitis. The authors advocated that treatment was indicated whenever this organism was isolated from the clinical specimens [15].

In another prospective study [16] of 15 patients with bacteremically proven Klebsiella pneumonia, right upper lobe involvement was documented in 11 cases. The authors observed that immunosupression was a significant risk factor for Klebsiella pneumonia and infections were mostly hospital acquired. Of the 15 patients, K. oxytoca was isolated in five, four of whom had bilateral disease. The authors stated that $K$. oxytoca was seen more often in patients with bilateral infiltrates while $K$. pneumoniae was more common in patients with unilateral infiltrates [16]. Our patient too had right upper lobe necrotising pneumonia with infiltrates in the left lung. $K$. oxytoca has also been implicated as an offending agent for hypersensitivity pneumonitis [17].

The chronic form of the disease caused by Klebsiella species is rarely encountered. In the pre-antibiotic era, Solomon [1] reviewed 17 patients with chronic Friedlander pneumonia and documented that it occurred predominantly in males in the later decades and especially those affected by alcoholism. Upper lobe lesions were seen in $14(82 \%)$ patients and the involvement of multiple lobes occurred in 5 (30\%). Lung abscesses were documented in majority of these patients. Both lungs were involved in only two patients. The author further stated that frequent occurrence of blood streaking and mucopurulent sputum along with the cavities in upper lobes on chest X-ray can lead to strong suspicion and even treatment for pulmonary tuberculosis in these patients [1].

A study published from Japan [18] reported four patients with Klebsiella (Friedlander's) pneumonia of whom, one had a chronic presentation with typical cavitary lung abscesses in the right lung [18]. Chronic presentation of pneumonia due to $K$. pneumoniae has also been reported from India in an immunocompetent elderly male. He was a reformed smoker who, prior to presentation, had received anti-tuberculous therapy with no relief due to marked clinical and radiological resemblance to that of pulmonary tuberculosis. Subsequently, a diagnosis of chronic Klebsiella pneumonia was established when initial sputum samples cultured K. pneumoniae. The patient had a marked symptomatic improvement with appropriate antibiotics and was largely symptom free. A chest X-ray done after eight months showed considerable clearing of the lesion with some remaining fibrosis [11]. Imaging in our current patient too had a marked resemblance to that of pulmonary tuberculosis as there was cavitation in the right upper lobe.

Non-resolving pneumonia of 9 months duration caused by $K$. oxytoca has been documented 
only once before from Canada in an 82-year-old previously healthy woman with a 30 pack-year history of smoking. Radiologically, the patient had an extensive consolidation of the left lower lobe with volume loss, which was confirmed on HRCT along with emphysematous changes. The diagnosis was established with the culture of the organism from bronchoalveolar lavage [14]. In our patient too, non-resolving pneumonia along with a history of smoking and emphysematous changes were present. K. oxytoca was cultured as a pure growth from both sputum samples as well as the bronchial aspirate.

The chronic presentation of pneumonia caused by $K$. pneumoniae is an uncommon clinical entity while that due to $K$. oxytoca is a distinct rarity as it has been documented only once before. Physicians, especially in high tuberculous prevalent areas, should be aware that the chronic form of the disease due to Klebsiella species can have a presentation akin to that of pulmonary tuberculosis.

\section{Conflict of interest}

The authors declare no conflict of interest.

\section{References:}

1. Solomon S. Chronic Friedlander infections of the lung. JAMA 1940; 115: 1527-1536.

2. Craven DE, Steger KA. Epidemiology of nosocomial pneumonia. New perspectives on an old disease. Chest 1995; 108 (2 Suppl.): 1S-16S.

3. Marston BJ, Plouffe JF, File TM Jr et al. Incidence of community-acquired pneumonia requiring hospitalization. Results of a population-based active surveillance Study in Ohio. The
Community-Based Pneumonia Incidence Study Group. Arch Intern Med 1997; 157: 1709-1718.

4. Bartlett JC. Bacterial pneumonia. In: Gorbach SL, Bartlett JG, Blacklow NR (ed.). Infectious diseases; $2^{\text {nd }}$ ed. Philadelphia: WB Saunders Company 1998: 571-582.

5. Hug BL, Rossi M. A year's review of bacterial pneumonia at the central hospital of Lucerne, Switzerland. Swiss Med Wkly 2001; 131: 687-692.

6. Bansal S, Kashyap S, Pal LS, Goel A. Clinical and bacteriological profile of community acquired pneumonia in Shimla, Himachal Pradesh. Indian J Chest Dis Allied Sci 2004; 46: 17-22.

7. American Thoracic Society; Infectious Diseases Society of America. Guidelines for the management of adults with hospital-acquired, ventilator-associated, and healthcare-associated pneumonia. Am J Respir Crit Care Med 2005; 171: 388-416.

8. Chung DR, Song JH, Kim SH et al. High prevalence of multidrug-resistant non fermenters in hospital-acquired pneumonia in Asia. Am J Respir Crit Care Med 2011; 184: 1409-1417.

9. Erasmus LD. Friedlander bacillus infection of the lung; with special reference to classification and pathogenesis. Q J Med 1956; 25: 507-521.

10. Prince SE, Dominger KA, Cunha BA, Klein NC. Klebsiella pneumoniae pneumonia. Heart Lung 1997; 26: 413-417.

11. Sinha R, Panjabi C, Varma M, Vijayan VK, Shah A. Chronic Klebsiella pneumonia in an immunocompetent host. J Assoc Physicians India 2003; 51: 306-308.

12. Colebunders R, Bastian I. A review of the diagnosis and treatment of smear-negative pulmonary tuberculosis. Int J Tuberc Lung Dis. 2000; 4: 97-107.

13. Seaton D. Pneumonia. In: Seaton A, Seaton D, Leitch G (ed.). Crofton and Douglas's respiratory diseases; $5^{\text {th }}$ ed. London: Blackwell Science Ltd. 2000: 356-444.

14. Al-Moamary MS, Copland GM. Nonresolving pneumonia due to Klebsiella oxytoca: an unusual presentation. Clin Infect Dis 1998; 26: 765-766.

15. Power JT, Calder MA. Pathogenic significance of Klebsiella oxyto$c a$ in acute respiratory tract infection. Thorax 1983; 38: 205-208.

16. Korvick JA, Hackett AK, Yu VL, Muder RR. Klebsiella pneumonia in the modern era: clinicoradiographic correlations. South Med J 1991; 84: 200-204.

17. Kane GC, Marx JJ, Prince DS. Hypersensitivity pneumonitis secondary to Klebsiella oxytoca. A new cause of humidifier lung. Chest 1993; 104: 627-629.

18. Tsukadaira A, Okubo Y, Kobayashi $\mathrm{T}$ et al. Four cases of Klebsiella pneumonia. Nihon Kokyuki Gakkai Zasshi 2002; 40: $530-535$ 